\title{
Coordination Optimization of PSS Parameters based on Polymorphic Bacterial Chemotaxis Algorithm
}

\author{
Hongsheng $\mathrm{Su}$ \\ School of Automation and Electrical Engineering, Lanzhou Jiaotong University, \\ Lanzhou 730070, China \\ shsen@163.com
}

\begin{abstract}
In terms of damping low frequency oscillation, power system stabilizer(PSS) plays a very key role. However, in terms of PSS capability exploitation, it is quite important io select the suitable parameters assignment. Different from traditional bptimization algorithms with eigenvalue analysis based and with system damping ratio as the gim function, in this paper a new PSS parameters method based on the Polymorphic Bactenial Chemotaxis(PBC) algorithm is presented for PSS parameters optimi ation. According to the time multiplied absolute error integral criterion (ITAE criteria), this paper chooses the ability of tracking a given value with minimum error of system output as the objective function. Thus the whole process of the disturbed system is considered. Finally, Eigenvalue analysis and nonlinear time-domain simulation are conducted to verify the Jeasibility and effectiveness of the proposed method.
\end{abstract}

Keywords: Power system stabilizer, parameter optimization, Polymorphic Bacterial Chemotaxis algorithm, ITAE crtteria

\section{Introduction}

In recent years, China s power system has developed quite rapidly. Due to the expansion of the grid size, and the long-distance and weak connection of large regional power grids, and as well as the use of a variety of fast excitation systems, the low-frequency oscillation happens in such a system-sometimes. As a result, the safety and stability on electric power system is seriously influenced. Clearly, the most effective way to solve this problem is to retrofit power system stabilizer (PSS) excitation system.

If only installing PSS with appropriate parameters at the appropriate units can effectively damp the low frequency oscillation of the system, or this may enhance a certain oscillation modes, and deteriorate the damping of other modes at the same time, eventually can't improve the overall damping of the system, and even worsen the effect. Hence, for the whole system al-sided coordination optimization of PSS parameters is required.

With the interconnection of large area power grid and the application of high gain and rapid excitation devices, the low frequency oscillation phenomena have occurred in modern large area interconnected power system, which has a serious impact on the security and stability of the power grid. To install a PSS in the generator's excitation system is the most cost and effective way to damp low-frequency oscillations[1]. The PSS provides an additional amplitude damping torque to suppresses low-frequency oscillation by compensation of the phase lag which caused by rapid excitation system. To select the reasonable parameters of the PSS is the foundation of playing a damping role. 
In actual power system, the PSS parameters design is based on experience and site standalone experiment, but the diverse interconnected units in the grid have mutual influence in the practical system, the parameters designed for standalone unit are unable to reach optimal or near optimal. As the actual operation of power grid is changing every time, it is necessary to optimize all PSS parameters of the system on the basis of preliminary design so that the added additional damping controllers can consider both generator sets and power grid operation to adapt to the specific operation of power system and improve the effect in a large power grid. In recent studies, a variety of smart controllers have been proposed, for instance, adaptive power system stabilizer, and fuzzy logic power system stabilizers, and as well as power system stabilizer based on neural network[2-4], and et al., But the traditional PSS with fixed structure and parameters is still the widely used controller in power system, it has great practical significance to select and optimize their parameters.

Presently, the scholars at domestic and foreign have been using a variety of optimization algorithms to perform PSS parameters optimization, such as simulated annealing algorithms, and tabu search algorithms, and genetic algorithms[5-7]. They have played a certain effect in PSS parameters optimization, but they still explore some defects in maneuverability and convergence. In this paper, the Polymorphic Bacterial Chemotaxis(PBC) algorithm is proposed to optimize the PSS parameters as it possesses stronger local and global search capabilities, and is quite efficient and requires the less population numbers. In [8] several multidimensional functions is tested using the $\mathrm{PBC}$ algorithm, the results show that the $\mathrm{PBC}$ algorithm's calculation quantity is less than several other populay algorithms, and its stability is very excellent as it can get the optimal solution every time. The PBC algorithm has already been successfully used to solve the feed forward weights of neural network in [9], and the prediction of stock price in [10], and the image registration of sensor in [11]. As a new intelligent algorithm, PBC algoritnm, shows better performance than any other similar algorithms, and is worth conducting further research and application.

\section{Mathematics Model}

\subsection{Model Description}

In this paper the IEEE ST1 excitation system is selected to serve as the experiment object with traditjonal PSS [12], which can be shown in Figure 1.

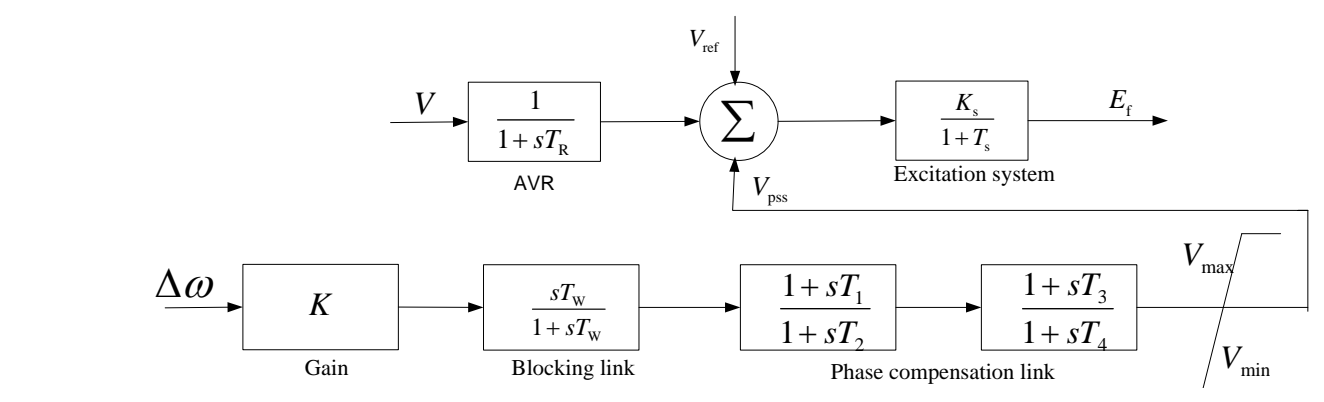

Figure 1. IEEE ST1 Excitation System with PSS

Seen from Figure 1, PSS includes the three links, i.e., gain link, and DC blocking link, and phase compensation link. The speed deviation signal $\Delta \omega$ is selected as the input signal of the PSS, and whose output signal $V_{\mathrm{pss}}$ is added to the automatic voltage regulator(AVR) as an additional damping signal. the main control link of excitation system is instead using a proportional controller with inertia link, where $K_{\mathrm{s}}$ is proportional amplification factor, and $T_{\mathrm{s}}$ 
is the inertia time constant, $V$ is generator terminal voltage, and $V_{\text {ref }}$ is a reference voltage. Usually, PSS parameters of $T_{\mathrm{w}}$, and $T_{2}$, and $T_{4}$ take experienced values, such that, they are known, only the parameters $K, T_{1}$, and $T_{3}$ requires to be optimized.

\subsection{Aim Function and Constraints}

To define the appropriate aim function plays a critical role in damping low frequency oscillation effectively using the PSS. Generally speaking, in the problem of PSS parameter optimization, the selection of the objective function can be broadly divided into two categories. A method to construct aim function is to apply information provided by timedomain simulation results $[13,14]$, and another one is the characteristic values analysis method based on the linear model of the system, namely, it can use eigenvalues of system state matrix which can react system damping conditions to construct evaluation index [15].

In $[13,14]$, the transient kinetic energy is selected as the evaluation indicator of optimization effect of the PSS parameters as system transient kinetic energy and potential energy after clearing the fault is constant, that is, the transient energy is conserved corresponding to the system energy during oscillation. White using time-domain simulation method, it is necessary to set artificial disturbance so as to obtain the aim function. Although the disturbance is random, it is impossible for haman disturbances to stimulate all the oscillation modes of the system.

In [15-17], the indicators of small signal stability level of system are obtained through the eigenvalues of electromechanical oscillation-mode. Refêrences [5, 15] make the system weakest damping mode the maximum as ôptimization aim. It improves the system's weakest mode greatly, but cannot reach the overalleffect of optimization. In $[6,16]$ the optimization goal is to maximize the sum damping of system electromechanical oscillation mode. The method considers the small signal stability level of the entire system, and so it increases damping of each oscillation mode, but did noconsider allocating damping between each mode. According to the principle of conservation of the damping of the system, it may result in improving damping of conte modes, at the same time deteriorating damping of certain modes.

In former studies, most of themrchoose the minimum electromechanical mode damping of the system as aim function of PSS parameter optimization. In order to be able to take into account the output error of gystem both during the oscillation process and the end of oscillation process [17], the paper puts the rotor angular deviation as tracking target, and sets the capacity that systemoutput tracks the given value with minimum error as the optimization objective function, that is, ITAE criteria, the objective function is written by.

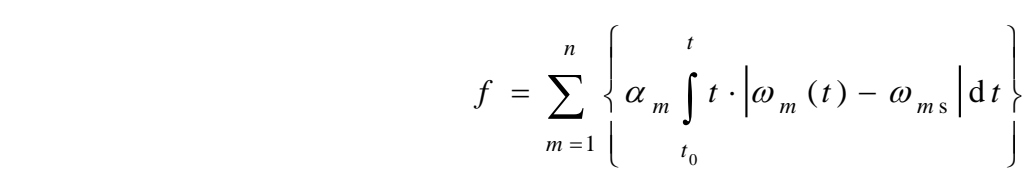

where $n$ is the number of units, and $\omega_{m}(t), \omega_{m s}$ are the practical value and steady-state one of rotor angular speed of the $m$ th unit, respectively, and $\alpha_{m}$ is the weight factor of the diverse units.

PSS parameter coordination optimization problem is transformed into the following optimization issue.

$$
\min f(\boldsymbol{R})
$$


where $\boldsymbol{R}$ is a vector which is composed of the parameters to be optimized.

Let the range of the parameters to be optimized serve as the constraint conditions of the optimization problem, which can be described below.

$$
\left\{\begin{aligned}
K_{\text {min }} & \leq K_{k} \leq K_{\text {max }} \\
T_{1, \text { min }} & \leq T_{1, k} \leq T_{1, \text { max }} \\
T_{3, \text { min }} & \leq T_{3, k} \leq T_{3, \text { max }}
\end{aligned}\right.
$$

where $k$ is the number of units.

According to experience, the range of $K_{k}$ is in range of $[0.01,100]$, and the range of $T_{1, k}$ and $T_{3, k}$ is in [0.01, 1], and the values of $T_{\mathrm{w} k}, T_{2 k}$, and $T_{4 k}$, are usually given in advance

To avoid the issue of quality solutions annihilation during the processing of the traditional constraints with improper penalty function, we take another way to handle constraints by introducing the following transformation.

$$
r_{j}=\frac{r_{j, \mathrm{~min}}+r_{j, \mathrm{max}}}{2}+\frac{r_{j, \mathrm{~min}}-r_{j, \mathrm{max}}}{2} \sin \left(w_{j}\right)
$$

where $\boldsymbol{r} \in \boldsymbol{R}$.

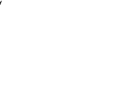

We then use a new vector $\boldsymbol{x}=\left(x_{2}, \cdots, x_{3}, \cdots, x_{n}\right)$ to instead the previous vector $\boldsymbol{R}=\left(r_{1}, r_{2}, \cdots, r_{j}, \cdots, r_{m}\right)$. And thus, the above constrained optimization problem is transformed into an unconstrained optimization problem as follows.

$$
\text { (2) } \min f(\boldsymbol{X})
$$

\section{PBC Algorithm and Improvement}

\subsection{Bacterial Colony Chemotaxis Algorithm}

In recent years, as anew bionic evolution algorithm, bacterial colony chemotaxis (BCC) algorithm was proposed in [18]. The algorithm simulates the reaction mechanism of bacteria action under the environment attractant. It is different from the pure random search of genetic algorithm(GA) with the fitness function. The bacteria can make use of its own location information to determine the movement direction of next step, and so it is a gradient approximation search method.

The following assumptions require to be conducted on the movement of single bacteria. The locus of the bacteria is composed by a series of lines, and each rail line is described by three indicators: the speed, direction and duration. The bacteria mobile search procedure is described below.

For convenient analysis, we firstly establish three system parameters related to the algorithm precision $\varepsilon$, that is, $T_{0}$, and $t_{c}$, and as well as $b$.

$$
T_{0}=\varepsilon^{0.30} \cdot 10^{-1.73}
$$




$$
\begin{gathered}
b=T_{0} \cdot\left(T_{0}^{-1.54} \cdot 10^{0.60}\right) \\
t_{c}=\left(b / T_{0}\right)^{0.31} \cdot 10^{1.16}
\end{gathered}
$$

where $T_{0}$ is the smallest average moving time, and $t_{\mathrm{c}}$ is relevant time, and $\mathrm{b}$ is system parameter.

(1) Assume that all the tracks have the same constant speed, here let it equal 1.

(2) To calculate the moving time $t$ of the bacteria in the new track. Assume that $t$ follows the exponential probability density distribution, that is,

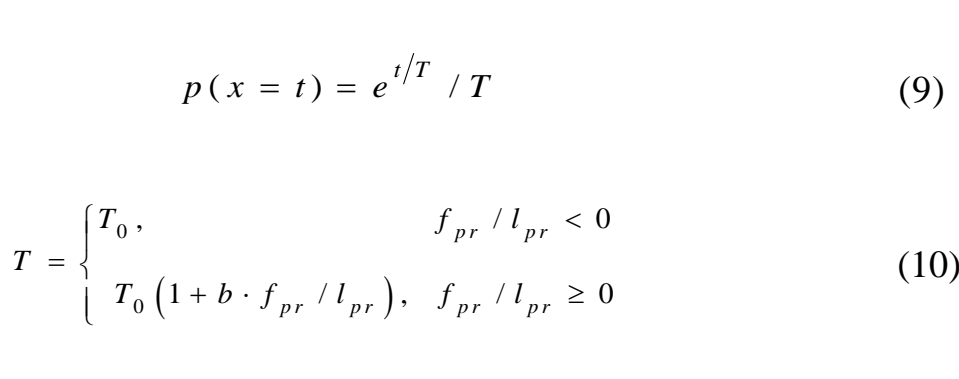

where $f_{\mathrm{pr}}$ is the difference between the function value of current position and the value of previous position, $l_{\mathrm{pr}}$ is the vector modulus connecting the current position and the previous position, $b$ is a system parameter.

(3) To calculate the new moving direction and moving distance of bacteria. Here the moving distance is described by $r$, and the moving direction is $\boldsymbol{\Psi}=\left(\varphi_{1}, \varphi_{2}, \cdots, \varphi_{i}, \cdots, \varphi_{n-1}\right)$ of $n$-1 dimension angle vector, where $\varphi_{i}$ is a head angle of new direction opposite to the original direction in the $i$ th dimension, and $\varphi_{i}$ follows the Gaussian probability distribution according to moving left or right.

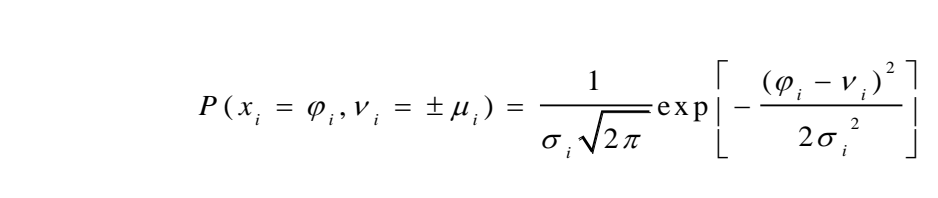

In (11), the expected value $\mu$ and variance $\delta$ can be calculated using the following formula.

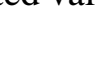

$$
\begin{cases}\mu=1.0821\left(1-e^{-t_{\mathrm{c}} t_{\mathrm{pr}}}\right) \quad \sigma=0.4538\left(1-e^{-t_{\mathrm{c}} t_{\mathrm{pr}}}\right), & f_{\mathrm{pr}} / l_{\mathrm{pr}}<0 \\ \mu=1.0821 & \sigma=0.4538,\end{cases}
$$

where is the duration time in the previous track.

The moving step-length vector $\overrightarrow{\boldsymbol{L}}=\left(l_{1}, l_{2}, \cdots, l_{i}, \cdots, l_{n}\right)$ in new track can be calculated via $r$ and $\Psi$ from a polar to Cartesian coordinate transformation.

$$
l_{1}=r \cdot \prod_{k=1}^{n-1} \cos \varphi_{k}
$$




$$
\begin{gathered}
l_{i}=r \cdot \sin \varphi_{i-1} \cdot \prod_{k=1}^{n-1} \cos \varphi_{k}, \quad i=2,3, \cdots n-1 . \\
l_{n}=r \cdot \prod_{k=1}^{n-1} \sin \varphi_{k}
\end{gathered}
$$

(4) To calculate bacteria's new position. Let the location of the bacteria be $\vec{X}=\left(X_{1}, X_{2}, \cdots, X_{i}, \cdots, X_{n}\right)$, and the new location of the bacteria is then expressed by

$$
\overrightarrow{\boldsymbol{X}}_{i, k+1}=\overrightarrow{\boldsymbol{X}}_{i, k}+\overrightarrow{\boldsymbol{L}}_{k}
$$

\subsection{Bacterial Perception Process}

Before every time movement, the bacteria firstly percept the surrounding environment and probe whether there are other bacteria staying at better position. If so, the bacteria will tend to move to the center point of the bacteria which have a better position. When the moving number is $k$, the center point of the bacteria which have a betteposition near the $i$-th bacteria is calculated by the following formula(for example, we will seek the minimum value of the function).

$$
\operatorname{Center}\left(\overrightarrow{\mathrm{X}}_{i, k}\right)=\operatorname{Aver}\left(\overrightarrow{\mathrm{X}}_{j, k} \mid f\left(\mathrm{X}_{i, k}\right) \overrightarrow{\mathrm{X}}_{f, k}\right.
$$

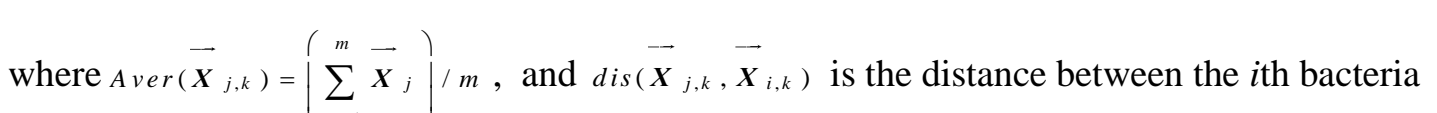

and the jth bacteria, and Senselimit is the perception range of bacteria, and $m$ is the number of bacteria which have a better position in terms of the $i$ th bacteria.

The mobile track line length can be described below.

$$
\operatorname{rand}(0,2) \cdot \operatorname{dis}\left(\overrightarrow{\boldsymbol{X}}_{i, k}, \text { Center }\left(\overrightarrow{\boldsymbol{X}}_{j, k}\right)\right)
$$

where $\operatorname{ran}(0)$, is a random value between $(0,2)$.

\subsection{BCC Algorithm Improvement}

(1) In the iterative process, we can adaptively update the convergence precision progressively according to the following formula.

$$
\varepsilon_{\text {new }}=\varepsilon_{\text {old }} / \alpha
$$

where $\alpha$ is a constant which can be used to update the calculation accuracy. 
(2) The perception range can be adjusted, dynamically. To do this, we define the fitness variance of the population as follows.

$$
\sigma^{2}=\sum_{i=1}^{n} \frac{\left(f_{i}-f_{\mathrm{av}}\right)^{2}}{f_{\mathrm{s}}}
$$

where $f_{i}$ is the fitness value of the $i$ th bacteria, and $f_{\mathrm{av}}$ is the average fitness value of the bacterial flora, and $f_{\mathrm{s}}$ is the standard value of fitness value difference, which is calculated using the following formula.

The perception range of the $k$-th step is

$$
f_{\mathrm{s}}=\left\{\begin{array}{cc}
\max \left\{\left|f_{i}-f_{\mathrm{av}}\right|\right\}, & f_{i}-f_{\mathrm{av}} \neq 0 \\
1, & f_{i}-f_{\mathrm{av}}=0
\end{array}\right.
$$

In the above formula, $S_{\max }$ and $S_{\min }$ respectively express the maximum perception value and the minimum one.

\subsection{Multi-group PBC Algorithm}

BCC algorithm adds information sharing mechanism within the population, and however, the overall location updating strategy is still quite simple. In [11] a polymorphic bacterial chemotaxis(PBC) algorith was proposed based on the BCC algorithm, here PBC algorithm is analogous to the multi-groups like other similar intelligent optimization algorithm, such as Niche algorithm, polymorphic ant colony algorithm (PACA), multi-particle swarm algorithm(IPPSO) and et al. Bacteria are divided into several sub-flora and various flora forage independently. If there are multiple flora in a specified distance $D_{\min }$, we can compare their maximum fitness value, and then let these bacteria with a lower fitness value move so that the flora in the ent re search space can keep a certain distance between one another. By this method, the algorthm can maintain the diversity of the flora and avoid falling into the local optimum so that it has stronger searching capability in terms of the global optimization.

Group feeding and feeding between groups are complementary and indispensable. Foraging vithin one group is a local search in variable scale on the basis of a single flora, the purpose is to improve the ability of bacteria to find the extreme point through intensive search and decelerating the search speed to reduce the count amount. Foraging between groups more emphasizes global search on the basis of different types of flora, whose aim is to maintain the diversity of the flora and to inhibit degradation phenomena to prevent the algorithm falling into a local minimum value. In this paper, we combine the advantages of the two searching mechanisms of group foraging and foraging between groups to expect to be able to achieve a satisfying result.

These steps to optimize the PSS parameters applying PBC algorithm are described below.

(1) To initialize the positions of the diverse bacteria, and distribute bacterial population in different locations, randomly, and scatter it according to the range of variable. 
(2) To determine the initial convergence precision $\varepsilon$ and maximum number of iteration times $N_{\max }$, and then to calculate the system parameters of $T_{0}$ and $b$ and $t_{c}$ from (6) to (8).

(3) To calculate the center of the bacteria which are in better positions near the $i$ th one, and also the distance to need to move to the center, and then to calculate new location $\overrightarrow{\boldsymbol{x}}_{i, k+1}$.

(4) To compare the fitness value $f\left(\overrightarrow{\boldsymbol{X}}_{i, k+1}^{\prime}\right)$ with the fitness value ${ }_{f\left(\overrightarrow{\boldsymbol{X}}_{i, k+1}\right)}$ of the new location $\overrightarrow{\boldsymbol{X}}_{i, k+1}^{\prime \prime}$ that the bacteria to be moved to before sense environment, if $f\left(\overrightarrow{\boldsymbol{X}}_{i, k+1}^{\prime}\right)<_{f\left(\overrightarrow{\boldsymbol{X}}_{i, k+1}^{\prime \prime}\right)}$, the bacteria move to the new position $\overrightarrow{\boldsymbol{X}}_{i, k+1}$, and move to $\overrightarrow{\boldsymbol{X}}_{i, k+1}$ otherwise.

(5) When the number of steps reaches the specified number $N_{\text {step }}$, if there are two sub-flora and the distance between them is less than the specified range, we then compare their maximum fitness value and define the position of bacteria with the maximum degree as the optimal solution.

(6) To repeat steps (3)-(5) until it meets abort conditions or the maximam number of iterations is reached.

The full algorithm flow chart is shown in Figure 2.

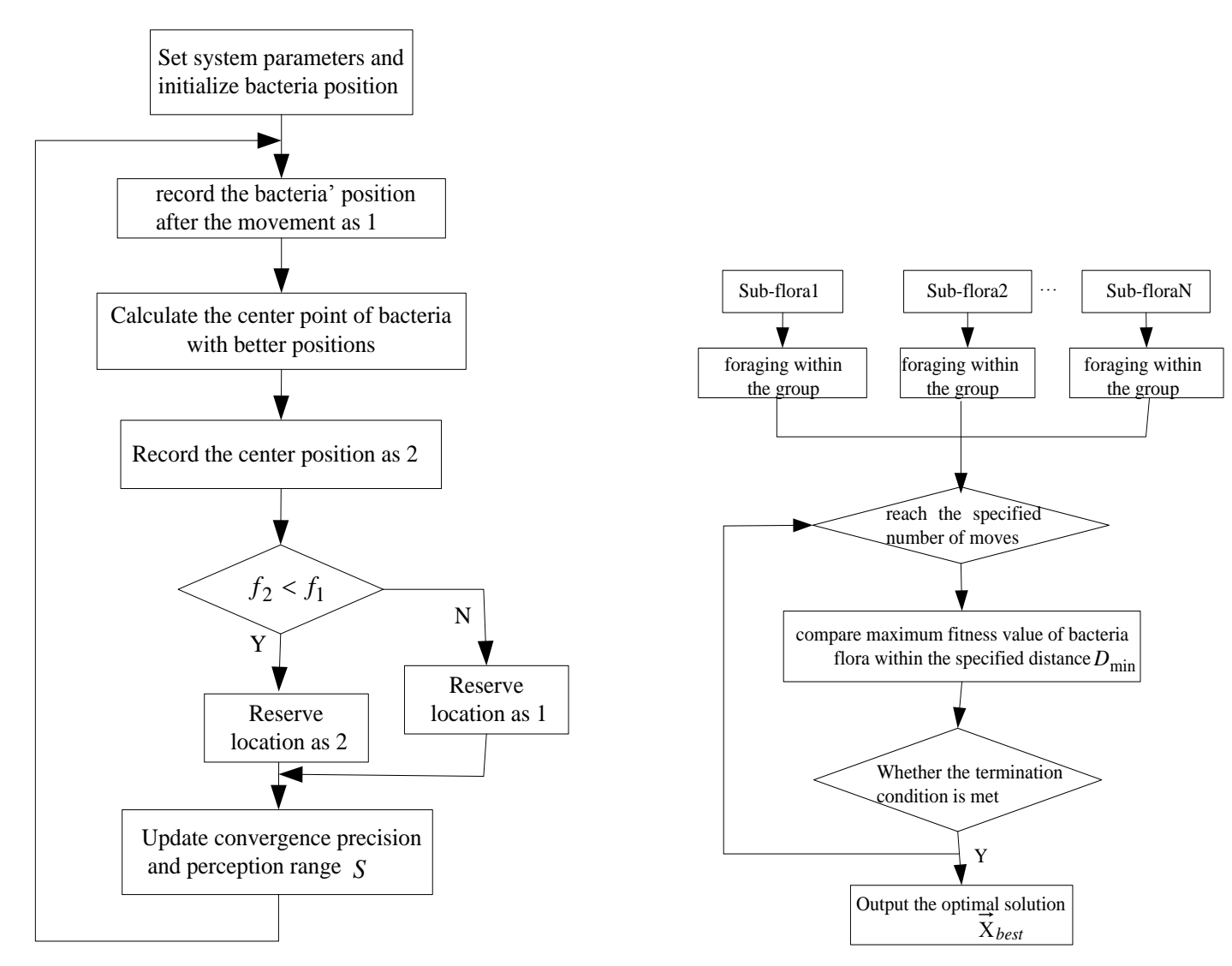

(a) Single BCC Algorithm Flow Chart

(b) Multi-colony Algorithm Flow Chart

Figure 2. PBC Algorithm Flow Chart 


\section{PSS Parameters Optimization Using PBC Algorithm}

Figure 3 shows the USA WSCC system [21], which contains three generators. Let the reference power value be $\mathrm{SB}=100 \mathrm{MVA}$, and reference voltage value be $\mathrm{UB}=230 \mathrm{kV}$, and system frequency be $60 \mathrm{~Hz}$. Generator G1 is balance node, G2 and G3 are PV nodes, the remaining nodes are PQ nodes. All generators use biaxial model and self-shunt static excitation system. Excitation gain $\mathrm{Ka}=200$, the time constant $\mathrm{Ta}=0.01$. As PSS is not installed, the eigenvalues, and frequency, and damping of the system are shown in Table 1.

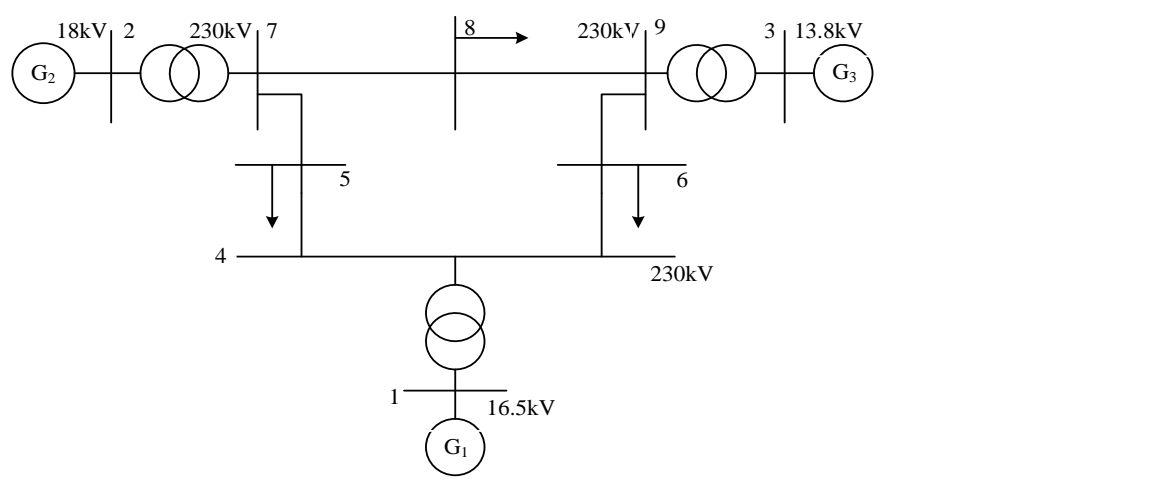

Figure 3. USA WSCC System Strugture

Table 1. System Electromechanical Mode before Installing PSS

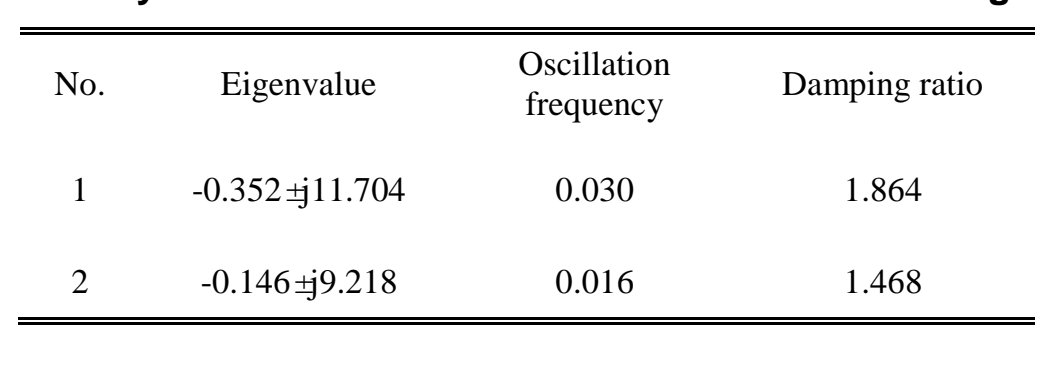

Seen from Table 1, the two pairs of the corresponding characteristic roots of the electromechanical oscillation mode are located near the left of the imaginary axis of the complex plane and is very close to the imaginary axis, inevitably, it makes the system damping ratio is smalland the system has very weak ability to suppress oscillations. The oscillation phenonenon can occur when the system operating mode is slightly altered. So it can't suppress the oscillation under large disturbance, thus the safe and stable operation of the system will be at risk. Therefore it is necessary to consider retrofitting PSS in this system in order to inprove the system damping and to prevent system oscillation.

According to participation factor method [22], we determine to install PSS on cell 1 and ceil 2, thus the parameters to be optimized are the gain $K_{1, k}$ and time constant $T_{1, k}, T_{3, k}$ of cell 1 and cell 2, where $k$ takes 1 or 2, the parameters of $T_{\mathrm{w}}, K_{2}$ and $K_{4}$ takes 20, 0.02, 0.02. Two units use the same weight $\alpha_{\mathrm{m}}=0.5$.

We define five flora in all, and each flora size is set as 10 with the dimension being 6 , and iteration times is 200 , and $D_{\min }=5$, and the initial accuracy is $\varepsilon_{\text {begin }}=2$, and the final accuracy is $\varepsilon_{\text {end }}=10^{-6}$. We can then write algorithm program and build simulation model in MATLAB7.0 environment. For convenience comparison, we respectively apply PBC and PSO algorithm to perform the PSS parameters optimization as shown in Table 2. 


\section{Table 2. Parameter Optimization Result}

\begin{tabular}{cccc}
\hline \hline & & $\mathrm{G}_{1}$ & \\
Algorithm & $K$ & $T_{1}$ & $T_{3}$ \\
PSO & 10.540 & 0.117 & 0.273 \\
PBC & 24.443 & 0.134 & 0.196 \\
& & $\mathrm{G}_{2}$ & \\
PSO & 15.774 & 0.178 & 0.304 \\
PBC & 27.842 & 0.140 & 0.171 \\
\hline \hline
\end{tabular}

The small signal stability analysis is conducted for the system installed PSS with the optimized parameters, the system's characteristics of electromechanical oscillation mode are shown in Table 3.

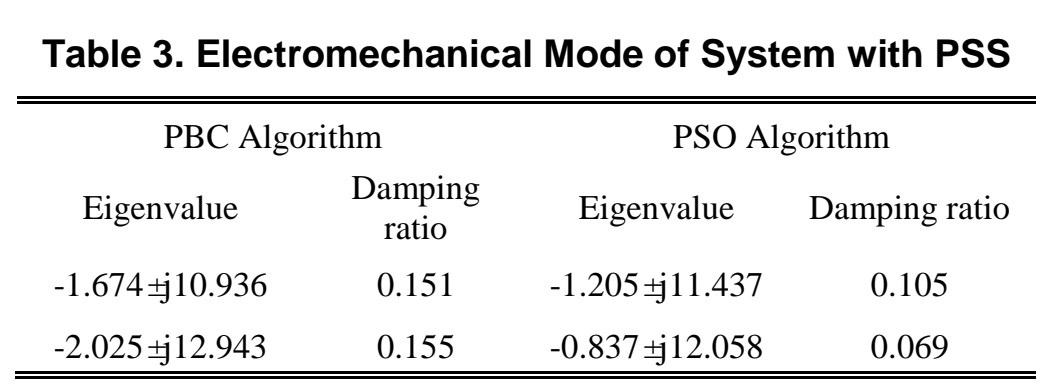

When PSS is not installed, modal analysis results show that the system possesses a weak damping, which can be seen from Table 1 . After installing PSS on units 1 and 2, the damping ratio of electromechanical oseillation mode has been significantly improved from Table 3. This shows the validity of PSS in suppressing low-frequency oscillation. At the same time it can be seen from Table 2 that the PSS with the optimized parameters by the PBC algorithm is better and the damping ratio have a significantly greater increase. From the actual optimization procedure, we also draw that search efficiency of PBC is higher than one of PSO algorithm, PBC algorithm is more sultable for applications in complex systems.

To further validate the response of the system installed PSS under large disturbance, we conduct dynamic time-domain simulation for the system. Let the disturbance of three-phase short circuit fault occurat 1.0s at the export of generator G1, and it continues for $0.1 \mathrm{~s}$. The power angle swing of C2, G3 to G1 are shown in Figure 4 and Figure 5.
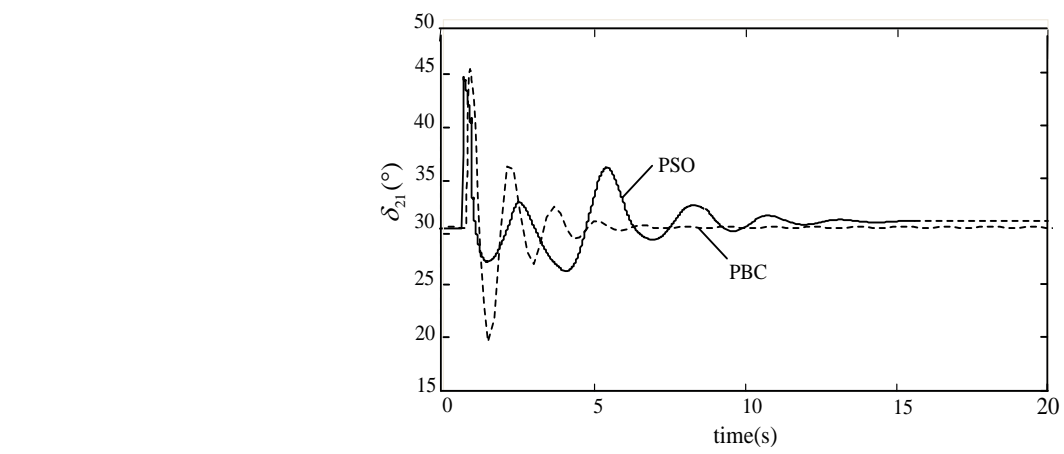

Figure 4. Power Angle Swing of G2 to G1 


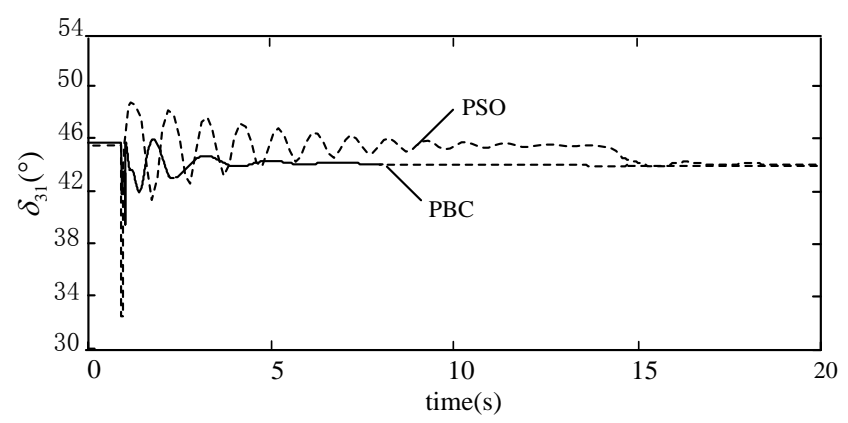

Figure 5. Power Angle Swing of G3 to G1

Seen from Figure 4 and Figure 5, the power angle swing between units which alopt improved PBC algorithm to optimize the parameters of the PSS is subsided within a shorter time, and so the PSS effect to suppress the system oscillation is better.

In another one example, IEEE 4-machine 11-node system in [21] is used to further test the algorithm proposed in this paper.

System's wiring diagram is described in Figure 6.

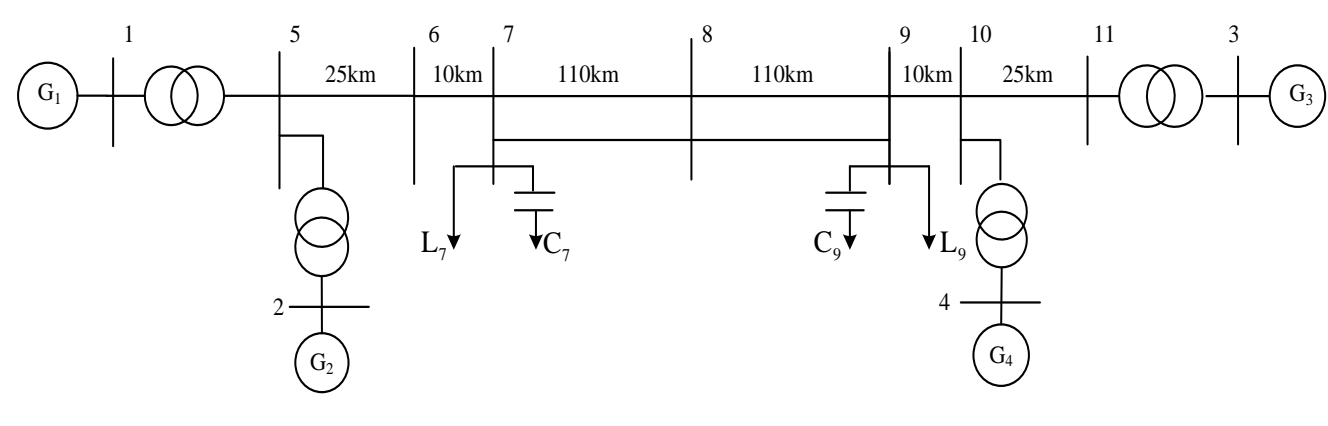

Figure 6. IEEE 4-machine 11-node System

The system is composed of wo areas where the generators G1, G2, G3, and G4 are connected by two long lines. The rated capacity of each cell is 900MVA, and the rated voltage is $20 \mathrm{kV}$ transmission system rated voltage is $230 \mathrm{kV}$. The first area transports 400MW power to the second area. All generators adopt fifth-order models, excitation system parameters, generator parameters, load data and reactive parameter values of capacitor can be found in $[15,16]$

4.

Before installing, PSS, the system eigenvalues, frequency and damping are shown in Table .

\section{able 4. System Electromechanical Mode before Installing PSS}

\begin{tabular}{cccc}
\hline \hline Electromechanical mode & Eigenvalue & Frequency & Damping ratio \\
& $+0.031 \pm$ & & \\
1 & $\mathrm{j} 3.84$ & 0.61 & -0.008 \\
& $-0.490 \pm$ & & \\
2 & $\mathrm{j} 7.15$ & 1.14 & 0.07 \\
& $-0.496 \pm$ & & 0.07 \\
\hline \hline
\end{tabular}


Seen from Table 4, the system possesses three electromechanical oscillation modes, where the mode 2 and mode 3 are local mode, and mode 1 is oscillation between areas with the negative damping, and so mode 1 oscillation is unstable.

From participation factor method [22], we install PSS on unit 2 and 4, so the parameters to be optimized are the gain $K_{1, k}$ and time constant $T_{1, k}, T_{3, k}$ of unit 2 and 4 , where $k$ takes 2 and 4, and $T_{\mathrm{w}}, K_{2}$ and $K_{4}$ takes 20, 0.02, 0.02. Two units use the same weight $\alpha_{\mathrm{m}}=0.5$.

We similarly set five flora, and each flora size is 10 , and its dimension is 6 , and evolution algebra is $200, D_{\min }=5$, and initial accuracy is $\varepsilon_{\text {begin }}=2$, and final accuracy is $\varepsilon_{\text {end }}=10^{-6}$. We can then write algorithm program and build simulation model in MATLAB7.0 environment. PSS parameters optimization results are shown in Table 5.

Table 5. Parameter of Optimization Results

\begin{tabular}{ccccccc}
\hline \hline \multirow{2}{*}{ Algorithm } & \multicolumn{4}{c}{ Unit $\mathrm{G} 2$} & \multicolumn{3}{c}{ Unit G4 } \\
& $K_{2}$ & $T_{12}$ & $T_{32}$ & $K_{4}$ & $T_{14}$ & $T_{34}$ \\
PSO & 28.360 & 0.183 & 0.169 & 30.784 & 0.164 & 0.172 \\
PBC & 31.472 & 0.214 & 0.267 & 29.863 & 0205 & 0.193 \\
\hline \hline
\end{tabular}

The optimized PSS is added to the system, and then the eigenvalues, and frequency, and as well as damping of electromechanical mode can be worked,outas shown in Table 6.

Table 6. ElectromechanicanMode of System with PSS

\begin{tabular}{cccc}
\hline Algorithm & Eigenvalue & $\begin{array}{c}\text { Parametes } \\
\text { Frequency }\end{array}$ & Damping ratio \\
& $-0.972 \pm \mathrm{j} 4.578$ & 0.73 & 0.208 \\
PSO Algorithm & $-3.520 \pm \mathrm{j} 12.436$ & 1.98 & 0.272 \\
& $-8.376 \pm \mathrm{j} 1.251$ & 1.79 & 0.440 \\
& $-1.382 \pm \mathrm{j} .584$ & 0.57 & 0.360 \\
PBC Algorithm & $-4.912 \pm \mathrm{j} 11.626$ & 1.85 & 0.389 \\
& $-0.353 \pm \mathrm{j} 11.748$ & 1.87 & 0.661 \\
\hline
\end{tabular}

From Table 6, we can see that after the system is installed PSS optimized by the PBC algorithm, all electromechanical mode eigenvalues are moved to the left of $S=-1$, and all damping ratio reach to upper to $30 \%$. The results are significantly better than one optimized by PSO algorithm and the system low-frequency oscillation is suppressed effectively.

In order to tesi dynamic performance of the system optimized, let the system suffer a large disturbance, that is, three-phase short-to-ground fault occurs in bus 8 at $1 \mathrm{~s}$ and the fault is cleared at $1.2 \mathrm{~s}$. And then we conduct time-domain simulation for the system. The simulation results are shown in Figure 7 to Figure 12.

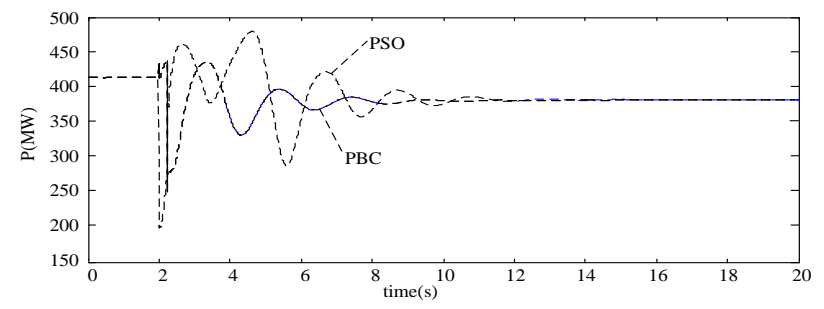

Figure 8. Power Fluctuation of bus7-9 after the Fault 


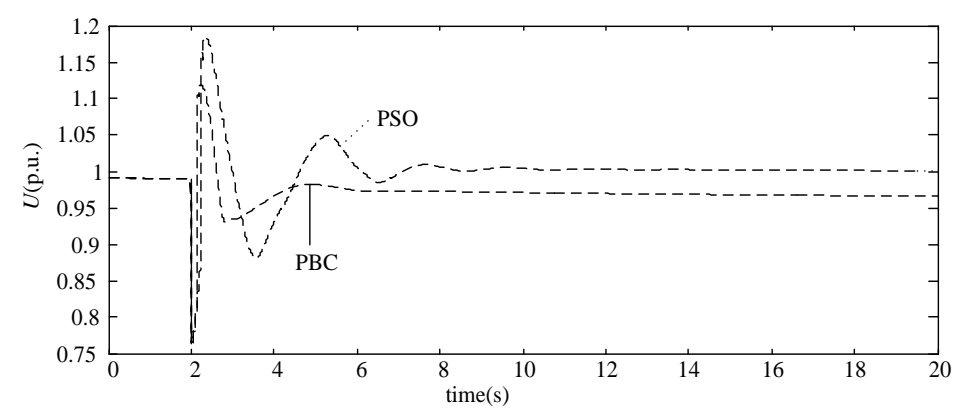

Figure 9. Voltage Fluctuation of bus7

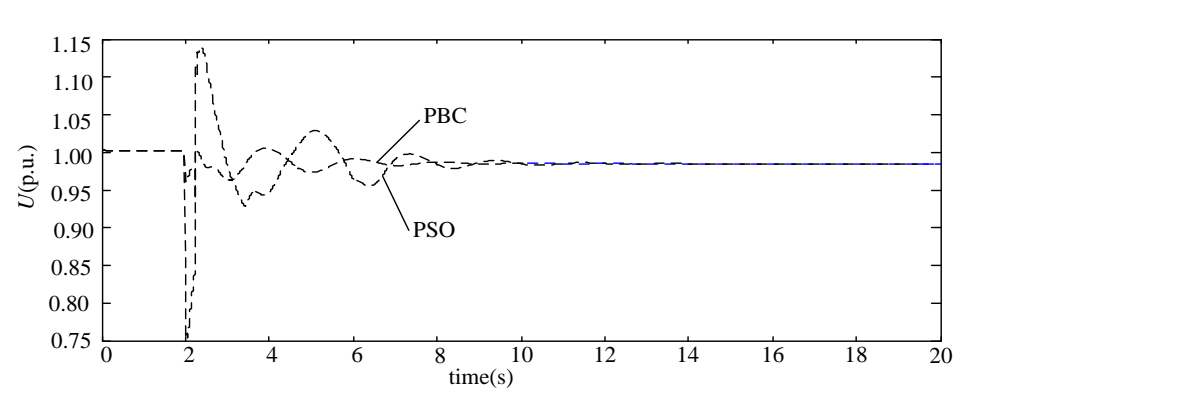

Figure 10. Voltage Fluctuation of bus 9

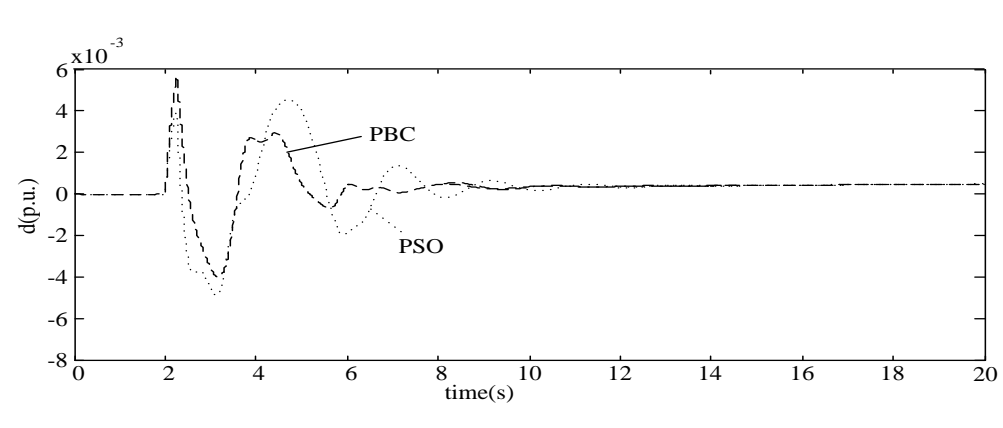

Figure 11. Rotor Speed Deviation of Generator 2 after the Fault

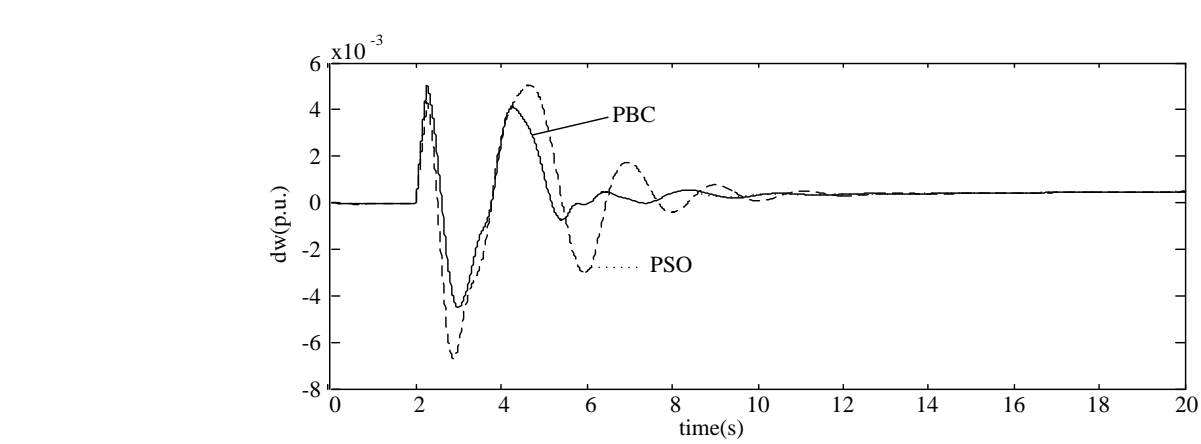

Figure 12. Rotor Speed Deviation of Generator4 after the Fault 


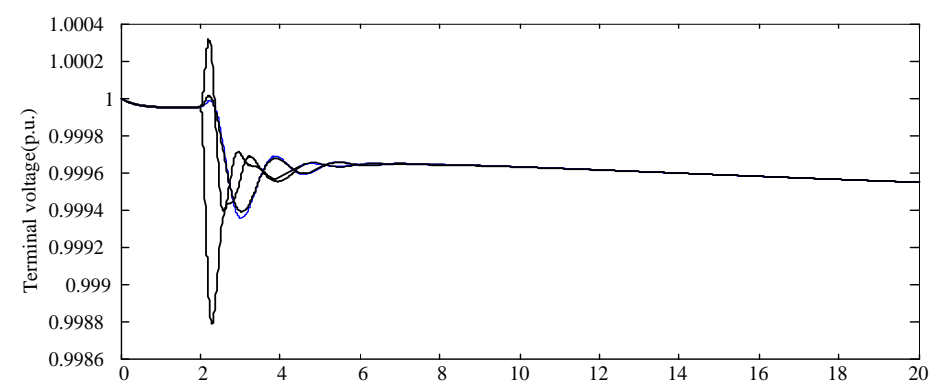

Figure 13. Terminal Voltage of 1-4 Unit

We can see from the above Figures that after the system is added the PSS optimized by the PBC algorithm, the voltage fluctuations of bus bar 7 and 9, and the active power fluctuations of bus bar 7-9, and angular velocity deviation tend to stabilize faster. The terminal voltage fluctuations of the units are limited in the allowable range and stabilized quickly. The PSS with optimized parameters by PBC algorithm improve the static and dynamic stability of the system better.

Above eigenvalue analysis and the three-phase short circuit fault simatlation test results show that the PSS optimized based on PBC optimization algorithn makes the system damping ratio significantly improved, and the rotor angular speed and the oscillation of the electromagnetic power after the fault have been well suppressed to keep the system stable. The optimization results of PBC algorithm are better than the traditional PSO algorithm.

\section{Conclusion}

This paper presents a new intelligent eptimization algorithm for PSS parameters coordination optimization in multi-machine power system, which is called as PBC based on Bacterial Chemotaxis algorithm. PBC algorithm not only retains the ability of bacteria individual to change the search directions using its own location information, but also combines information exchanging within the group between groups during searching. Hence it is of strong capability of locar search and global search. It is high efficient and only a smaller number of populations is required. So it is particularly suitable for large-scale optimization problems solying.)

The former researches on PSS parameters optimization are centered on eigenvalue analysis, and use system damping ratio as the objective function. Different from that, this paper chooses the ability of tracking a given value with minimum error of system output as the aim function, which considers the entire oscillation process of system from getting disturbed to tend to stabilize. To apply this method to select objective function can avoid the curse of dimensionality when calculating the eigenvalues. Eigenvalues analysis and dynamic simulation results show that the proposed method makes the system damping significantly improved and shows the effectiveness and superiority of the PBC algorithm, which is a novel interligent optimization algorithm, and possesses further study and application value. The method mentioned in the paper is also a useful supplement for the PSS parameters adjustment in reality.

\section{Acknowledgements}

This project was supported by the National Natural Foundation of China (Grant No.61263004) and Railways Ministry Science and Technology Research and Development Program (2012X003-B). 


\section{References}

[1] Y. X. Ni, S. S. Chen and B. L. Zhang, Editor, Theory and Analysis of Dynamic Power System, Tsinghua University Press, Beijing (2002).

[2] Z. Y. Tan, S. J. Cheng and W. Xuan, "A network based adaptive power system stabilizer", Automation of Electric Power Systems, vol. 24, no. 15, (2000), pp. 1-6.

[3] Y. W. Liang, Z. J. Hu and Y. P. Chen, "A power system stabilizer based on neural network inverse system", Transactions of China Electrotechnical Scoiety, vol. 19, no. 5, (2004), pp. 61-65.

[4] H. Zhao, L. Y. Liu and H. J. Wang, "Study of fuzzy power system stabilizer based on slide mode control", Power system technology, vol. 28, no. 12, (2004), pp. 9-11.

[5] M. A. Abido, "Parameter optimization of multi-machine power system stabilizers using genetic local search", International Journal of Electrical Power and Energy Systems, vol. 23, (2001), pp. 785-794.

[6] M. A. Abido, "A novel approach to conventional power system stabilizer design using Tabu search", International Journal of Electrical Power and Energy Systems, vol. 21, (1999), pp. 443-454.

[7] M. A. Abido, "Robust design of multi-machine power system stabilizers using simulated annealing", IEEEE Trans. Energy Conversion, vol. 15, no. 3, (2000), pp. 297-304.

[8] W. W. Li, H. Wang and Z. J. Zhou, "Function optimization method based on bacterial colony chemotaxis", Journal of Circuits and Systems, vol. 10, no. 1, (2005), pp. 58-63.

[9] Y. D. Zhang and L. N. Wu, "Weights optimization of neural network via improved BCO approach", Progress in Electromagnetic Research(PIER), vol. 83, (2008), pp. 185-198.

[10] Y. D. Zhang and L. N. Wu, "Stock market prediction via combination of improved BCO approach and BP neural network", Expert Systems with Applications(ESWA) vol 36, (2009), pp. 88498854.

[11] Y. D. Zhang, L. N. Wu, G. Wei and H. Q. Wu, "Auomatic sensor image registration via polymorphic bacterial chemotaxis", Computer Engineering and Applications, vol. 45, no. 4, (2009), pp. 30-33.

[12] S. L. Fang and Z. Fang, "Principle and Application of Power System Stabilizer", China Electric Power Press, Beijing, (1996).

[13] P. Zhang and A. H. Coonick, "Coordinated synthesi of PSS parameters in multi-machine power systems using the method of inequalities applied to genetic algorithms", (IEEE Transactions on Power Systems, vol. 15, no. 2, (2005), pp. 811-816.

[14] M. A. Pal, "Energy function analysis for power system stability, Kluwer Academic Publisher, New York, (1989).

[15] L. J. Cal and I. Erlich, "Simultaneous coordinated Uning of PSS and FACTS damping controllers in large power systems", IEEE Transactions on Power Systems, vol. 20, no. 1, (2005), pp. 294-300.

[16] D. Bomfim, AL. B. Taranto, G. N. and D. M. Falcao, "Simultaneous tuning of power system damping controllers using genetic algorithms", IEEE Transactions on Power Systems, vol. 15, no. 1, (2001), pp. 163-169.

[17] D. Y. Xue and Y Q. Chen MATLAB Solutions to Mathematical Problems in Control", Tsinghua University Press, Beijing, (2007).

[18] S. D. Muller, J. Marchetto, S, Airaghi and P. Koumoutsakos, "Optimization based on bacterial chemotaxis", IEEE Transaction of Evolutionary Computation, vol. 6, no. 1, (2002), pp. 16-29.

[19] P. Kundur, "Power System Stability and Control, Mc Graw-Hill, NewYork, (1994).

[20] X. F. Wang, W. L Fang and Z. C. Du, "Modern Power System Analysis", Science Press, Beijing, (2003).

[21]H. K. Dong and H. C. Jae, “Advances in Web Intelligence”, Springer-Verlag, (1994).

[22] G. H. Xu, Y. S. Su and Q. Sun, "Identification of PSS optimum location based on eigenvalue analysis method", Relay, vol. 32, no. 8, (2004), pp. 1-4.

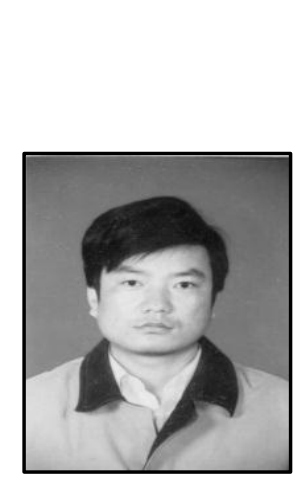

\section{Author}

Hongsheng Su, obtained his Master in Traffic Information Engineering and Control, Lanzhou Jiaotong University in 2001. He acquired his $\mathrm{PhD}$ in Power Systems and Its Automation, Southwest Jiaotong University. Now he is serving as a full-time professor at school of Automation and Electrical Engineering, Lanzhou Jiaotong University. His research interest includes System Security and Reliability, Intelligent Control, Power Systems and Its Automation, and etc. 
International Journal of Hybrid Information Technology

Vol.7, No.5 (2014)

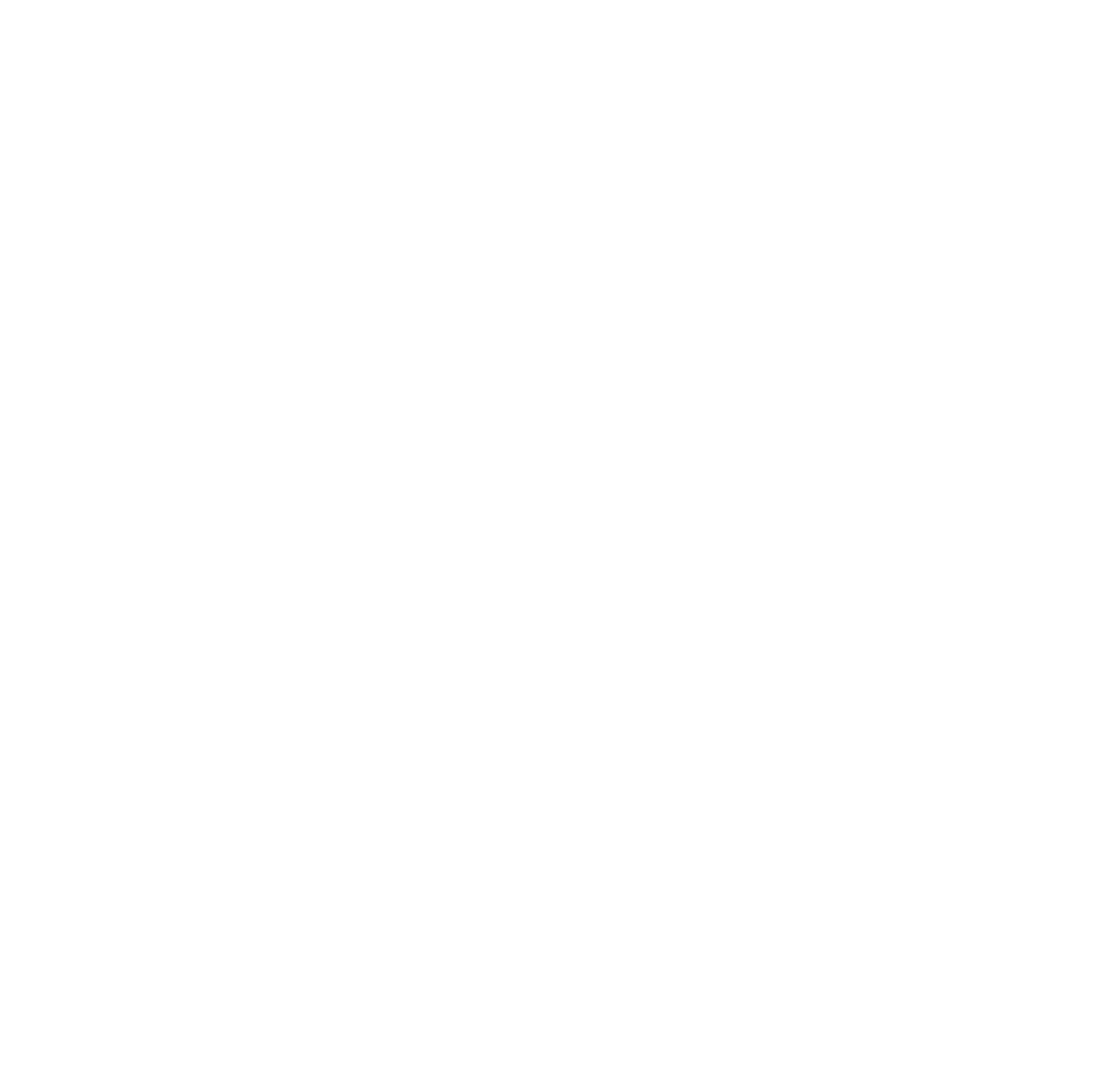

